\title{
Recurrence and 5-FU sensitivity of stage I/II node-negative breast, lung, or gastric cancer with occult neoplastic cells in lymph node sinuses
}

\author{
MASAYA MUKAI ${ }^{1}$, SHINKICHI SATO ${ }^{2}$, TAKAYUKI TAJIMA ${ }^{5}$, HIROMI NINOMIYA ${ }^{2}$, KANAKO WAKUI $^{2}$, \\ NOBUKAZU KOMATSU ${ }^{2}$, KAZUTOSHI TSUCHIYA ${ }^{3}$, HISAO NAKASAKI ${ }^{4}$ and HIROYASU MAKUUCHI ${ }^{5}$ \\ ${ }^{1}$ Department of Surgery, Tokai University Hachioji Hospital, Ishikawa-cho 1838, Hachioji, \\ Tokyo 192-0032; ${ }^{2}$ Department of Pathology, ${ }^{3}$ Endoscopy Center and ${ }^{4}$ Department of Surgery, \\ Tokai University Oiso Hospital, Gakyou 21-1, Oiso, Kanagawa 259-0198; ${ }^{5}$ Department of Surgery, \\ Tokai University School of Medicine, Bohseidai, Isehara, Kanagawa 259-1193, Japan
}

Received December 5, 2005; Accepted January 5, 2006

\begin{abstract}
This study was designed to examine the relationship between the presence of occult neoplastic cells (ONCs) in lymph nodes (LNs) and survival in 238 patients with stage I/II LN-negative cancer of the breast, lung, or stomach. In addition, immunohistochemistry for TS and DPD was used to compare the 5-FU sensitivity of the primary tumor in ONC (+) patients. The 5-year relapse-free survival (RFS) rate of 215 ONC (-) patients and 23 ONC (+) patients was 95.2 and $82.6 \%$, respectively $(\mathrm{p}=0.0107)$. The 5 -year overall survival (OS) rate of the ONC (-) and (+) patients was 97.4 and $77.4 \%$, respectively $(\mathrm{p}=0.0000)$. The $6 \mathrm{ONC}(+)$ patients with recurrence showed high and low TS expression in $33.3 \%$ $(2 / 6)$ and $66.7 \%(4 / 6)$, respectively, while high and low DPD expression was observed in $16.7 \%(1 / 6)$ and $83.3 \%(5 / 6)$, respectively. In the $17 \mathrm{ONC}(+)$ patients without recurrence, the corresponding values were $64.7 \%$ (11/17), $35.3 \%(6 / 17)$, $29.4 \%(5 / 17)$, and $70.6 \%(12 / 17)$. Patients with a combination of high TS and low DPD expression accounted for $33.3 \%$ $(2 / 6)$ of the ONC (+) patients with recurrence and $52.9 \%$ $(9 / 17)$ of those without recurrence, showing no significant
\end{abstract}

Correspondence to: Dr Masaya Mukai, Department of Surgery, Tokai University Hachioji Hospital, Ishikawa-cho 1838, Hachioji, Tokyo 192-0032, Japan

E-mail: mukai.masaya@hachioji-hosp.tokai.ac.jp

Abbreviations: ONCs, occult neoplastic cells; LN, lymph node; 5-FU, 5-fluorouracil; LV, leucovorin; TS, thymidilate synthase; DPD, dihydropyrimidine dehydrogenase; RFS, relapse-free survival; OS, overall survival; ITCs, isolated tumor cells

Key words: breast cancer, lung cancer, gastric cancer, occult neoplastic cells, chemosensitivity, 5-fluorouracil, thymidilate synthase, dihydropyrimidine dehydrogenase, micro-metastasis, isolated tumor cells difference between the two groups. These results suggest that ONCs are associated with a lower survival rate and that ONC (+) patients are unlikely to respond to 5-FU+LV therapy.

\section{Introduction}

Gastric cancer was the predominant cause of cancer death in Japanese men before the 1990s. With recent westernization of the diet and lifestyle, lung cancer has now become the leading cause of cancer death, followed by liver cancer and colorectal cancer (1). In Japanese women, however, gastric cancer is still the leading cause of cancer death, followed by colorectal cancer, lung cancer, and breast cancer (1). Stage I/II cancer of the breast, lung, or stomach without lymph node (LN) metastasis has been reported to cause life-threatening metastasis/recurrence in the liver or lungs of $5-15 \%$ of the patients who undergo curative resection (2-4). It is thought that potentially fatal hematogenous metastasis occurs in surgically treated patients when tumor cells enter the circulation during the perioperative period and survive to grow in a distant organ (5-7). Many studies have shown that the presence of immunohistochemically cytokeratin-positive occult neoplastic cells (ONCs) in LN sinuses distant from the primary tumor or the presence of circulating $\mathrm{ONC}$ clusters and malignant microaggregates is closely related to recurrence (8-18). ONCs can be counted with relative ease after immunostaining. Although these malignant cells cannot be detected in the portal or peripheral blood without complex techniques, ONCs can be identified by immunohistochemistry in the LNs protecting the host around the primary tumor (8-10). In the sixth version of the TNM classification, free cancer cells are classified as isolated tumor cells (ITCs), which are cell clusters less than $0.2 \mathrm{~mm}$ in diameter that are detectable by H\&E staining (19). However, it remains unclear how single cancer cells or clusters of just a few cells should be classified. In contrast, ONCs are defined as neoplastic cells floating in the sinuses of LNs distant from the primary tumor that are only detectable by cytokeratin immunostaining, including malignant microaggregates containing up to 10 cells (20). It has been reported 


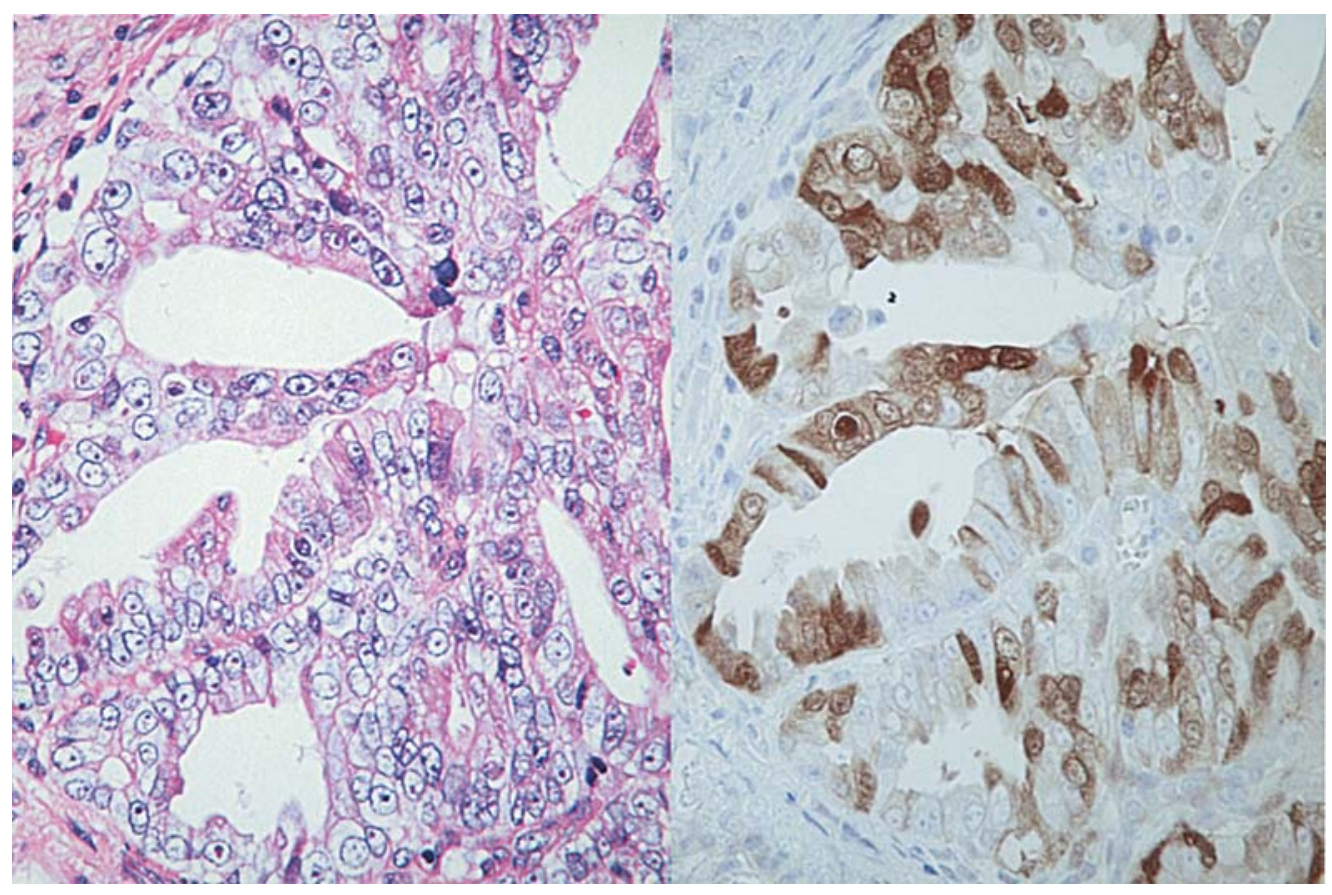

Figure 1. Immunohistochemical staining for thymidylate synthase (high expression; +++) in the primary gastric cancer of a stage II patient from the recurrence group with ONCs (left, H\&E staining, x200; right, TS stain, x200).

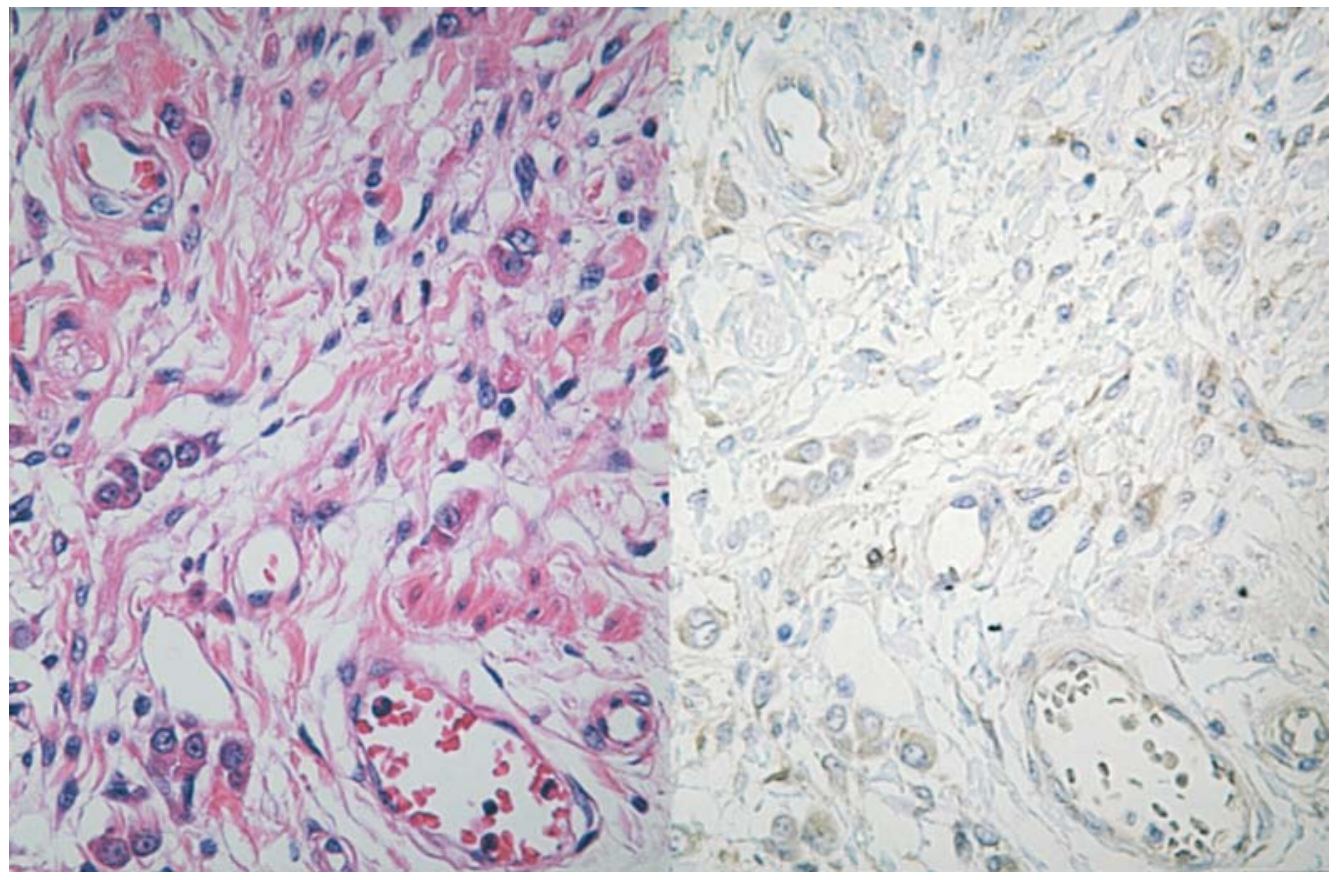

Figure 2. Immunohistochemical staining for dihydropyrimidine dehydrogenase (high expression; +++) in the primary gastric cancer of a stage II patient from the recurrence group with ONCs (left, H\&E staining, x200; right, DPD staining, x200).

that these cells may induce distant metastasis/recurrence in any organ of the body, and that ONCs should be differentiated from micrometastases anchored in LNs and should be considered to be a more aggressive form of occult systemic metastasis (20).

In the increasing number of patients with breast cancer or lung cancer, as well as colorectal cancer, the presence/absence of $\mathrm{LN}$ metastasis is known to be one of the most important prognostic indicators (2-5). LN metastasis has been extensively studied in patients with breast cancer, and it is considered possible to define whether this is a local or systemic disease from the presence/absence of metastasis, as well as the appropriate therapeutic methods and chemotherapy regimens (3). Oral preparations of 5-fluorouracil (5-FU) are available 
under the national health insurance scheme in Japan and are widely used to treat various types of cancer, including breast, lung, and gastric cancer without LN metastasis. These preparations are considered to be first-line postoperative adjuvant chemotherapy for stage II colorectal or gastric cancer (21-23). The mechanism by which 5-FU controls tumor growth involves FdUMP, a metabolite of 5-FU that forms a ternary complex with methylenetetrahydrofolate $\left(\mathrm{CH}_{2} \mathrm{FH}_{4}\right)$ and thymidylate synthase (TS), thereby inactivating TS and inhibiting DNA synthesis $(24,25)$. It is also known that more than $80 \%$ of each dose of $5 \mathrm{FU}$ is directly metabolized by dihydropyrimidine dehydrogenase (DPD) in the liver $(26,27)$. Therefore, TS and DPD are the target of 5-FU and are involved in its metabolism, respectively, making these enzymes important determinants of sensitivity to the anticancer effect of 5FU+LV therapy. Earlier studies have shown that tumors with high TS and low DPD expression are most sensitive to 5-FU+LV (24-27). However, no previous study used immunohistochemistry to assess the sensitivity of the primary tumor to $5-\mathrm{FU}$ in patients with stage I/II LNnegative breast, lung, or gastric cancer and ONCs in their regional LNs.

The present study was designed to examine the relationship between the detection of ONCs in LNs and the survival of patients with stage I/II LN-negative breast, lung, or gastric cancer. In addition, immunohistochemistry for TS and DPD was used to compare the 5-FU sensitivity of the primary tumor in ONC-positive patients.

\section{Materials and methods}

Among patients with LN-negative stage I/II primary breast, lung, or gastric cancer who underwent curative resection from 1987 to 2000,238 patients (76 with breast cancer, 24 with lung cancer, and 138 with gastric cancer) who met the following criteria were studied: i) patients with complete and reliable medical records who could be followed to assess recurrence and survival; and ii) patients with at least $10 \mathrm{LNs}$ that were negative on histological examination after routine $\mathrm{H} \& \mathrm{E}$ staining.

Detection of ONCs in LNs and calculation of the 5-year relapse-free survival (RFS) rate and 5-year overall survival $(O S)$ rate. Immunohistochemical detection of cytokeratin in LNs was performed by the indirect immunoperoxidase method using a monoclonal anti-cytokeratin antibody (AE1/AE3; Fuji Chemical Industries, Ltd., Japan) $(28,29)$. A total of 2,380 nodes were assessed in the 238 patients. Serial sections of each LN were stained with H\&E and keratin-AE1/AE3 to determine the positive rate and positive tumor cell count. Attached cancer cells and/or tumor cell nests growing in the LNs were excluded and only tumor cells floating freely in the sinuses were counted as ONCs. Immunostaining for cytokeratin was assessed as follows: detection of 3 or more cytokeratin-positive cells was defined as positive, while detection of 1 or 2 cytokeratin-positive cells was defined as negative (8-11). The data obtained by examining the LNs were used to classify the patients into ONC (+) and ONC (-) groups, and the 5-year RFS and 5-year OS rates were calculated for each group.
Immunohistochemical staining of the primary tumor for TS and DPD. Patients with ONCs in their LNs were divided into a group that developed metastasis/recurrence within 5 years (recurrence group) and a group without metastasis/recurrence for at least 5 years (non-recurrence group). Then the primary tumors of the patients in the recurrence and non-recurrence groups were immunohistochemically stained for TS and DPD by the indirect immunoperoxidase method using polyclonal anti-TS and -DPD antibodies (Taiho Pharmachemical Co. Ltd., Japan) as the primary antibodies (25-29). To assess the extent of immunostaining, stained tumor cells were counted at a high magnification in the entire maximum cut surface of the lesion. When no tumor cells were stained, the tumor was considered negative (-). When stained cells accounted for less than $5 \%$ of all tumor cells, the tumor was considered positive $(+)$. In addition, the tumor was classified as $(++)$ when stained cells ranged from $\geq 5 \%$ to $<30 \%$ of all tumor cells and as $(+++)$ when stained cells accounted for $\geq 30 \%$ of the total. The (-) or (+) tumors were classified as having a low level of TS or DPD expression, while $(++)$ or $(+++)$ tumors were classified as having high TS or DPD expression (Figs. 1 and 2) (21-23). Patients who had tumors with high TS and low DPD expression were defined as being more likely to respond to 5 FU+LV therapy.

Statistical analysis. To determine the 5-year RFS rate and 5 -year OS rate, survival was calculated by the Kaplan-Meier method and the log-rank test was used for comparison between the ONC (+) and ONC (-) groups. The $\chi^{2}$ test was also used to examine the significance of differences between the recurrence and non-recurrence groups. $\mathrm{P}<0.05$ was considered to indicate a significant difference in all analyses, which were performed using SPSS 13.0 J software (SPSS Japan, Inc., Tokyo, Japan).

\section{Results}

Of the 238 patients with LN-negative stage I/II breast, lung, or gastric cancer, 23 patients $(9.7 \%$, including 9 with breast cancer, 2 with lung cancer, and 12 with gastric cancer) were positive for ONCs and 215 patients $(90.3 \%$, including 67 with breast cancer, 22 with lung cancer, and 126 with gastric cancer) were negative for ONCs. The ONC (+) and ONC (-) patients had a 5-year RFS rate of $82.6 \%$ and $95.2 \%$, respectively ( $\mathrm{p}=0.0107$ ) (Fig. 3), while they had a 5-year OS rate of $77.4 \%$ and $97.4 \%$, respectively ( $\mathrm{p}=0.0000$ ) (Fig. 4).

The $23 \mathrm{ONC}(+)$ patients were divided into a recurrence group $(n=6)$ and a non-recurrence group $(n=17)$. The former group accounted for $26.1 \%$ of the ONC (+) patients $(6 / 23 ; 2$ with breast cancer, 1 with lung cancer, and 3 with gastric cancer) and the latter group accounted for $73.9 \%$ (17/23; 7 with breast cancer, 1 with lung cancer, and 9 with gastric cancer). Among the tumors of patients from the recurrence group, TS expression was high in $33.3 \%(2 / 6)$ and low in $66.7 \%(4 / 6)$, while DPD expression was high in $16.7 \%(1 / 6)$ and low in $83.3 \%$ (5/6) (Table I). Among the non-recurrence group, TS expression by the primary tumor was high in $64.7 \%$ $(11 / 17)$ and low in $35.3 \%$ (6/17), while DPD expression was high in $29.4 \%(5 / 17)$ and low in $70.6 \%(12 / 17)$ (Table I). Patients with a combination of high TS expression and low 


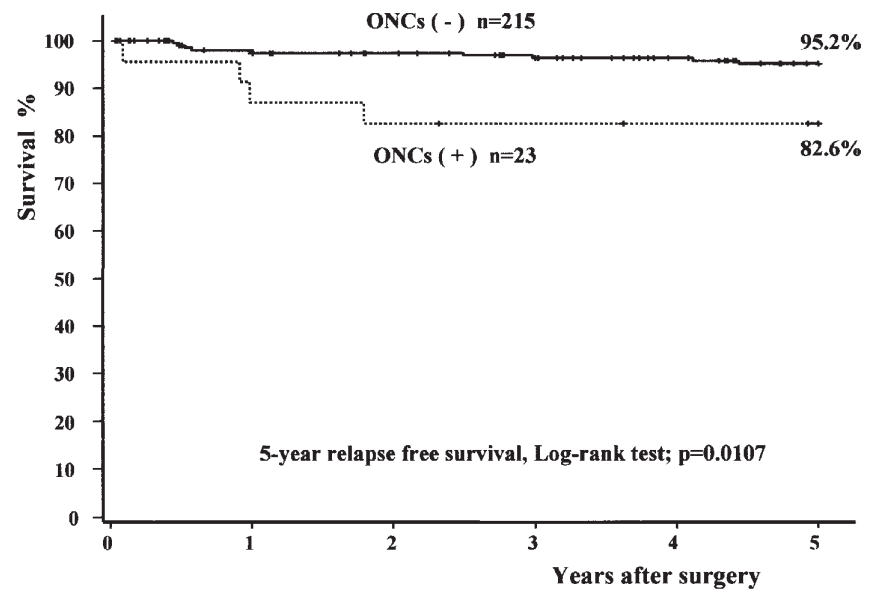

Figure 3. The 5-year relapse-free survival rate for patients with or without occult neoplastic cells (ONCs) among 238 patients with $\mathrm{LN}$-negative stage I/II breast, lung, or gastric cancer. The rate was $95.2 \%$ for 215 patients without ONCs and $82.6 \%$ for 23 patients with ONCs ( $\mathrm{p}=0.0107)$.

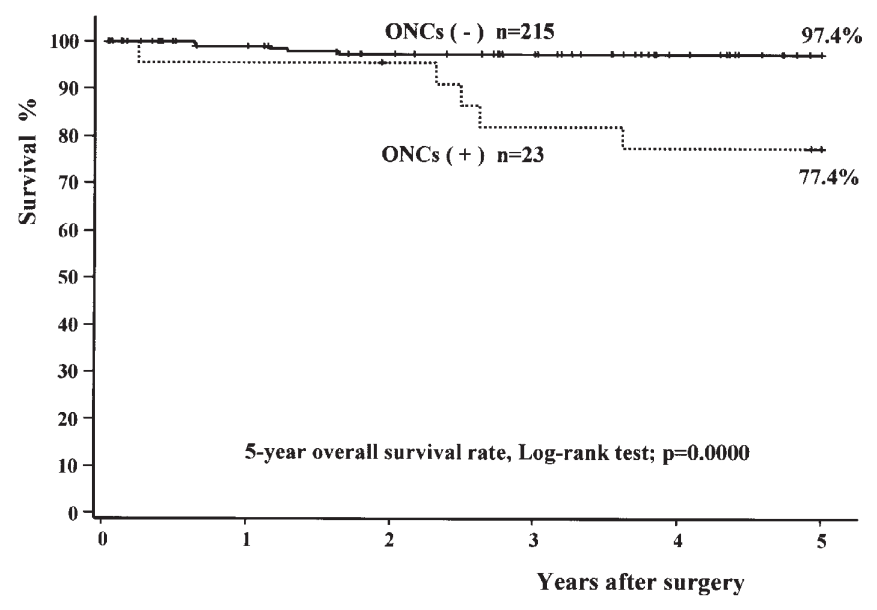

Figure 4. The 5-year overall survival rate for patients with or without occult neoplastic cells (ONCs) among 238 patients with LN-negative stage I/II breast, lung, or gastric cancer. The rate was $97.4 \%$ for 215 patients without ONCs and $77.4 \%$ for 23 patients with ONCs $(\mathrm{p}=0.0000)$.
DPD expression accounted for $33.3 \%(2 / 6)$ of the recurrence group and $52.9 \%(9 / 17)$ of the non-recurrence group, with no significant difference between the two groups (Table I).

\section{Discussion}

Based on currently available data, it is generally considered that LN-negative stage I or II cancer is unlikely to recur (1-4). However, there are no markers available to identify the patients in whom recurrence will occur. Earlier studies have shown that confirming the absence of ONCs can be used to identify patients who are unlikely to develop recurrence after the curative resection of LN-negative stage I/II cancer, since this method reliably identified a low-risk group with a specificity and negative predictive value of $90 \%$ or more, thus greatly reducing the mental burden on those patients $(12,15,16,18)$. Accordingly, the investigation of ONCs can be used for identifying a low-risk group rather than a high-risk group of patients with stage I/II LN-negative cancer. In the present study, however, the presence of ONCs had an adverse influence on survival, although the patients with ONCs only accounted for $9.7 \%$ of the total subject population (23/238). This may support the concept that occult systemic metastasis represented by circulating ONCs is more aggressive than micrometastasis and ITCs, which show a controversial relationship to survival at least partly due to their imprecise definitions (20).

Among the ONC (+) patients, 26.1\% (6/23) experienced recurrence. It is possible that they may have directly developed hematogenous metastasis/recurrence without an intermediate stage of perioperative LN metastasis. Stage I/II cancer without $\mathrm{LN}$ metastasis is often a regional disease limited to the primary tumor. However, a very small number of viable cancer cells are disseminated from the primary tumor throughout the body in patients with various types of cancer (11). Almost all of these malignant cells are probably eliminated by phagocytosis or other defenses when they invade peripheral lymphatics and/or vessels because the host immune system recognizes them as foreign $(6,7)$. It is therefore considered that the number of circulating malignant cells that escapes the host defenses is too small to directly induce metastasis/

Table I. Expression of thymidylate synthase (TS) and dihydropyrimidine dehydrogenase (DPD) in the primary tumors of 23 patients with stage I/II LN-negative breast, lung or gastric cancer and occult neoplastic cells (ONCs) in their lymph node sinuses. ${ }^{\text {a }}$

Immunohistochemical staining [high-level, (+++) (++); low-level (+) (-)] TS DPD TS and DPD

\begin{tabular}{|c|c|c|c|c|c|}
\hline \multirow[b]{2}{*}{ Total 23 cases } & & & \multirow[b]{2}{*}{ High-TS/low-DPD } \\
\hline & High-level & Low-level & High-level & Low-level & \\
\hline Recurrence $(n=6)$ & $33.3 \% \quad(2 / 6)$ & $66.7 \%(4 / 6)$ & $16.7 \%(1 / 6)$ & $83.3 \% \quad(5 / 6)$ & $33.3 \%(2 / 6)$ \\
\hline Non-recurrence $(n=17)$ & $64.7 \%(11 / 17)$ & $35.3 \%(6 / 17)$ & $29.4 \%(5 / 17)$ & $70.6 \%(12 / 17)$ & $52.9 \%(9 / 17)^{\mathrm{b}}$ \\
\hline
\end{tabular}

an the recurrence group $(\mathrm{n}=6)$ and the non-recurrence group $(\mathrm{n}=17)$, expression was classified as low $(-,+)$ or high $(++,+++)$. TS, thymidylate synthase; DPD, dihydropyrimidine dehydrogenase; ${ }^{b}$ not significant. 
recurrence, unless these cells can multiply in the favorable microenvironment of a distant organ. It is also possible that malignant microaggregates, a potential source of micrometastases, may escape from the primary tumor and reach a distant organ (20). For example, hematogenous metastasis/ recurrence of stage I/II LN-negative cancer may occur when malignant microaggregates derived from the primary tumor are trapped by the first filtering organ, such as the liver or lungs, after entering the circulation (20).

In general, the recurrence of gastric, breast, or lung cancer often involves metastasis to the liver and/or lungs, and these lesions are unlikely to be treated by surgical resection compared with metastases from colorectal cancer (21-23). The mode of recurrence of these cancers may depend on the aggressiveness of each tumor or organ-specific factors, including the growth potential of individual malignant microaggregates, but the most important factor may be the frequency at which viable cell clusters are released into the circulation from the capillary network around the primary tumor. Recent studies have shown that detection of circulating tumor cells in the peripheral blood provides an important indicator of the prognosis in patients with metastatic breast cancer $(30,31)$. In contrast, it is thought that ONC clusters and malignant microaggregates have already reached the target organs and are no longer in the peripheral blood during the perioperative period in patients with stage I/II cancer, so it may be important to detect and eliminate such clusters among free cancer cells (20-23).

The sensitivity of circulating ONCs, particularly malignant microaggregates, to anticancer drugs is important in relation to optimizing the dosages and chemotherapy regimens. For postoperative adjuvant chemotherapy to inhibit metastasis/ recurrence after curative resection of the primary tumor, it is necessary to eliminate residual cancer cells that have the potential to spread from the primary throughout the body during the perioperative period. Accordingly, it may be most effective to perform consolidation chemotherapy early after surgery. Therefore, it may also be necessary to directly examine the chemosensitivity of floating ONCs at the cellular level rather than measuring the activity of TS and DPD in tumor homogenates or immunostaining the primary tumor. It has generally been reported that TS is strongly positive in the germinal centers of non-neoplastic lymph nodes, while DPD is not detected $(25,27)$. However, the negative LNs of our cancer patients only showed weak staining for TS. Our preliminary study on the staining of floating ONCs revealed that neither TS nor DPD positivity could be assessed reliably. Since it was considered impracticable to prepare serial sections for H\&E, AE1/3, TS, and DPD staining from LNs measuring from several millimeters to approximately $1 \mathrm{~cm}$ in size and use these sections to assess the staining of individual ONCs at a cellular level, it was decided to investigate the TS and DPD expression by the primary tumors instead.

In the present study, oral fluoropyrimidine preparations were administered to $66.7 \%(4 / 6)$ of the ONC (+) patients with recurrence. Two of these 4 patients $(50 \%)$ had a combination of high TS and low DPD expression in their tumors, which is considered to be associated with a high sensitivity to 5-FU+LV therapy. Although the number of patients studied was very small, the chemotherapy regimen seemed to be inadequate in 2 of our 4 patients. Among the ONC (+) patients without recurrence, $58.8 \%(10 / 17)$ received oral fluoropyrimidine preparations, and $70 \%$ of them (7/10) showed a combination of high TS and low DPD expression, so the therapy was considered pharmacologically suitable for these patients. Earlier studies have shown that improving the survival rate of patients with recurrent cancer is most important to obtain a true survival benefit $(32,33)$. It may be more effective to identify stage I/II LN-negative patients with ONCs as a possible high-risk group after curative resection and start appropriate treatment at an early stage to eliminate circulating ONCs and malignant microaggregates during the perioperative period when occult systemic metastasis may precede overt recurrence. Such treatment should be possibly performed with the best combination of standard chemotherapy agents for each type of cancer to achieve a sufficient effect, although it will be necessary to collect more data in order to confirm the appropriate chemotherapy regimens for high-risk patients with stage I/II cancer.

\section{Acknowledgements}

This study was supported by grants from the Occult Neoplastic Cells Research and Study Group (\#2004-2291; Tokai University Hachioji Hospital, Hachioji, Tokyo, Japan) and the Research and Study Program of Tokai University Educational System General Research Organization (\#2005-48; Tokai University Hospital, Isehara, Kanagawa, Japan).

\section{References}

1. Journal of Health and Welfare Statistics, Health and Welfare Statistics Association, Tokyo 51: 46-49, 2004.

2. Omejc M, Juvan R, Jelenc F and Repse S: Lymph node metastases in gastric cancer: Correlation between new and old UICC TNM classification. Int Surg 86: 14-19, 2001.

3. Goldhirsch A, Glick JH, Gelber RD, Coates AS and Senn H-J: Meeting highlights: International consensus panel on the treatment of primary breast cancer. J Clin Oncol 19: 3817-3827, 2001.

4. Prenzel KL, Mönig SP, Sinning JM, Baldus SE, Brochhagen H-G, Schneider PM and Hölscher AH: Lymph node size and metastatic infiltration in non-small cell lung cancer. Chest 123: 463-467, 2003.

5. Mukai M, Ito I, Mukoyama S, Tajima T, Saito Y, Nakasaki H, Sato $\mathrm{S}$ and Makuuchi H: Improvement of 10-year survival by Japanese radical lymph node dissection in patients with Dukes' B and C colorectal cancer: A 17-year retrospective study. Oncol Rep 10: 927-934, 2003.

6. Mukai M, Tajima T, Nakasaki H, Sato S, Ogoshi K and Makuuchi H: Efficacy of postoperative adjuvant oral immunochemotherapy in patients with Dukes' B colorectal cancer. Ann Cancer Res Ther 11: 201-214, 2003.

7. Mukai M, Tajima T, Nakasaki H, Sato S, Ogoshi K and Makuuchi H: Efficacy of postoperative adjuvant oral immunochemotherapy in patients with Dukes' C colorectal cancer. Ann Cancer Res Ther 11: 215-229, 2003.

8. Mukai M, Sato S, Komatsu N, Nishida T, Shiba K, Ito I, Nakasaki $\mathrm{H}$ and Makuuchi $\mathrm{H}$ : Correlation between occult neoplastic cells in the lymph node sinuses and recurrence in patients with Dukes' C colorectal cancer. Oncol Rep 10: 1165-1169, 2003.

9. Mukai M, Sato S, Komatsu N, Nishida T, Shiba K, Ito I, Nakasaki $H$ and Makuuchi $H$ : Correlation between occult neoplastic cells in the lymph node sinuses and recurrence in patients with curatively resected Dukes' B colorectal cancer. Oncol Rep 10: 1177-1181, 2003.

10. Mukai M, Sato S, Nishida T, Komatsu N, Shiba K, Nakasaki H and Makuuchi H: Selection criteria for high risk and low risk groups of recurrence and metastasis in patients with primary colorectal cancer. Oncol Rep 10: 1753-1758, 2003. 
11. Mukai M, Sato S, Nakasaki H, Saito Y, Nishiumi N, Iwasaki M, Tokuda Y, Ogoshi K, Inoue H and Makuuchi H: Occult neoplastic cells in the lymph node sinuses and recurrence of primary breast, lung, esophageal and gastric cancer. Oncol Rep 11: 81-84, 2004.

12. Mukai M, Sato S, Komatsu N, Kimura T, Ninomiya H, Nakasaki H, Ogoshi K and Makuuchi M: Accuracy of criteria for predicting the recurrence and metastasis of stage I and II gastric cancer without lymph node metastasis. Oncol Rep 12: 59-62, 2004.

13. Mukai M, Sato S, Komatsu N, Kimura T, Ninomiya H, Nakasaki H, Ogoshi K and Makuuchi H: Accuracy of criteria for predicting recurrence and metastasis in stage II and III gastric cancer with lymph node metastasis. Oncol Rep 12: 63-66, 2004.

14. Mukai M, Sato S, Komatsu N, Kimura T, Ninomiya $\mathrm{H}$, Nakasaki H, Ogoshi K and Makuuchi H: Predicting the recurrence/metastasis of stage II and III breast cancer with lymph node metastasis. Oncol Rep 12: 303-306, 2004.

15. Mukai M, Sato S, Komatsu N, Kimura T, Ninomiya H, Nakasaki H, Ogoshi K and Makuuchi H: Predicting the recurrence/metastasis of stage I and II breast cancer without lymph node metastasis. Oncol Rep 12: 745-748, 2004.

16. Mukai M, Sato S, Tajima T, Kimura T, Komatsu N, Ninomiya H, Nakasaki H, Ogoshi K and Makuuchi H: Predicting recurrence and metastasis of stage II/Dukes' B colorectal cancer without lymph node metastasis. Oncol Rep 12: 1127-1130, 2004.

17. Mukai M, Sato S, Tajima T, Kimura T, Komatsu N, Ninomiya H, Nakasaki H, Ogoshi K and Makuuchi $\mathrm{H}$ : Predicting recurrence and metastasis of stage III/Dukes' C colorectal cancer with lymph node metastasis. Oncol Rep 12: 1301-1304, 2004.

18. Mukai M, Sato S, Tajima T, Kimura T, Komatsu N, Ninomiya H, Nakasaki H, Ogoshi K and Makuuchi H: Predicting recurrence and metastasis of Dukes' A primary colorectal cancer with or without proper muscle invasion. Oncol Rep 12: 1305-1308, 2004.

19. TNM Classification of Malignant Tumours. 6th edition. John Wiley \& Sons, Inc., New York, 2002.

20. Mukai M: Occult neoplastic cells and malignant micro-aggregates in lymph node sinuses: Review and hypothesis. Oncol Rep 14: $173-175,2005$.

21. Mukai M, Sato S, Ninomiya H, Wakui K, Komatsu N, Tsuchiya K, Nakasaki $\mathrm{H}$ and Makuuchi $\mathrm{H}$ : Recurrence and 5-FU sensitivity of stage III/Dukes' C colorectal cancer with occult neoplastic cells in lymph node sinuses. Oncol Rep 14: 1165-1169, 2005.

22. Mukai M, Sato S, Ninomiya H, Wakui K, Komatsu N, Tsuchiya K, Nakasaki $\mathrm{H}$ and Makuuchi H: Recurrence and 5-FU sensitivity of stage II/Dukes' B colorectal cancer with occult neoplastic cells in lymph node sinuses. Oncol Rep 14: 1171-1176, 2005.
23. Mukai M, Sato S, Ninomiya H, Wakui K, Komatsu N, Tsuchiya K, Nakasaki $\mathrm{H}$ and Makuuchi H: Recurrence and 5-FU sensitivity of node-positive stage II/III gastric cancer with occult neoplastic cells in lymph node sinuses. Oncol Rep 14: 1505-1510, 2005.

24. Berger SH and Hakala MT: Relationship of dUMP and free FdUMP pools to inhibition of thymidylate synthase by 5fluorouracil. Mol Pharmacol 25: 303-309, 1984.

25. Okabe H, Tsujimoto $\mathrm{H}$ and Fukushima M: Preparation of the antibodies against recombinant human thymidylate synthase for the detection of its intratumoral levels and application to sensitivity-study of 5-fluorouracil. Oncol Rep 4: 685-690, 1997.

26. Takenoue T, Kitayama J, Takei Y, Umetani N, Matsuda K, Nita ME, Hatano K, Tsuruo T and Nagawa H: Characterization of dihydropyrimidine dehydrogenase on immunohistochemistry in colon carcinoma and correlation between immunohistochemical score and protein level or messenger RNA expression. Ann Oncol 11: 273-279, 2000.

27. Kamoshida S, Shiogama K, Matsuoka H, Matsuyama A, Shimomura R, Inada K, Maruta M and Tsutsumi Y: Immunohistochemical demonstration of dihydropyrimidine dehydrogenase in normal and cancerous tissues. Acta Histochem Cytochem 36; 471-479, 2003.

28. Nakane PK and Pierce GB: Enzyme-labeled antibodies: preparation and application for localization of antigens. $J$ Histochem Cytochem 14: 929-931, 1966.

29. Nakane PK and Pierce GB: Enzyme-labeled antibody for the light and electron microscopic localization of tissue antigens. J Cell Biol 33: 307-318, 1967.

30. Cristofanilli M, Budd GT, Ellis MJ, Stopeck A, Matera J, Miller MC, Reuben JM, Doyle GV, Allard WJ, Terstappen LWMM and Hayes DF: Circulating tumor cells, disease progression and survival in metastatic breast cancer. N Engl J Med 351: 781-791, 2004.

31. Cristofanilli M, Hayes DF, Budd GT, Ellis MJ, Stopeck A, Reuben JM, Doyle GV, Matera J, Allard WJ, Miller MC, Fritsche HA, Hortobagyi GN and Terstappen LWMM: Circulating tumor cells: a novel prognostic factor for newly diagnosed metastatic breast cancer. J Clin Oncol 23: 1420-1430, 2005.

32. Feinstein AR, Sosin DM and Wells CK: The Will Rogers phenomenon: stage migration and new techniques as a source of misleading statistics for survival in cancer. N Engl J Med 312: $1604-1608,1985$.

33. Bunt AMG, Hermans J, Smit VTHBM, van de Velde CJH, Fleuren GL and Bruijn JA: Surgical/pathologic stage migration confounds comparisons of gastric cancer survival rates between Japan and Western countries. J Clin Oncol 13: 19-25, 1995. 